\title{
Commentary: Tricuspid valve ring characteristics: Physiologically important, clinically relevant, or too little too late?
}

\author{
Gabriel S. Aldea, MD
}

From the Department of Cardiothoracic Surgery, University of Washington Medical Center, Seattle, Wash. Disclosures: Author has nothing to disclose with regard to commercial support.

Received for publication Sept 25, 2019; revisions received Sept 25, 2019; accepted for publication Sept 25, 2019; available ahead of print Oct 9, 2019.

Address for reprints: Gabriel S. Aldea, MD, William K. Professor and Chief of Cardiac Surgery, Department of Cardiothoracic Surgery, University of Washington, 1959 NE Pacific St, Seattle, WA 98195-6310 (E-mail: aldea@uw.edu).

J Thorac Cardiovasc Surg 2021;161:e209-10

$0022-5223 / \$ 36.00$

Copyright (C) 2019 by The American Association for Thoracic Surgery

https://doi.org/10.1016/j.jtcvs.2019.09.133

Functional (secondary) tricuspid valve regurgitation (FTR) is the most common $(>85 \%)$ clinical presentation of significant tricuspid regurgitation (TR) but is a poorly characterized and understood set of overlapping conditions. Precise quantification and correct anatomic and physiologic diagnoses leading to more systematic, durable, and effective approaches remain elusive. ${ }^{1-3}$ FTR is a covariant with many known comorbid conditions (such as older age, lower ejection fraction, right ventricular [RV] dysfunction and dilatation, pulmonary hypertension, mitral regurgitation, left ventricular dysfunction, liver and renal dysfunction, and atrial enlargement and fibrillation) but is independently associated with a nearly 2 -fold increase in mortality. ${ }^{4,5}$ TR severity has to be assessed in conjunction with tricuspid valve (TV) annular size, TV morphology (leaflet tethering, chordal abnormalities, etc), RV size, function,and pulmonary vascular resistance. ${ }^{2}$ Treating concomitant TV dilatation and TR at the time of mitral valve surgery has been shown to be effective, is associated with minimal added morbidity and mortality, ${ }^{6-8}$ and currently meets class I and IIa indications for therapy in both American and European guidelines. Current therapies rely on TV annuloplasty devices with varied characteristics such as size, shape, form (planar and non-planar), and rigidity. There is also an increased recognition that in addition to annular therapies, severe leaflet tethering, when present, may also need to be addressed with additional interventions (such as leaflet augmentation, neochords, or subannular papillary muscle therapies). Because of historically high mortality for isolated FTR interventions, there remains significant uncertainties as to appropriate patient selection, timing, scope of repair, with yet-to-be-determined effectiveness, and durability of such approaches. This condition, understandably, remains undertreated. ${ }^{9}$

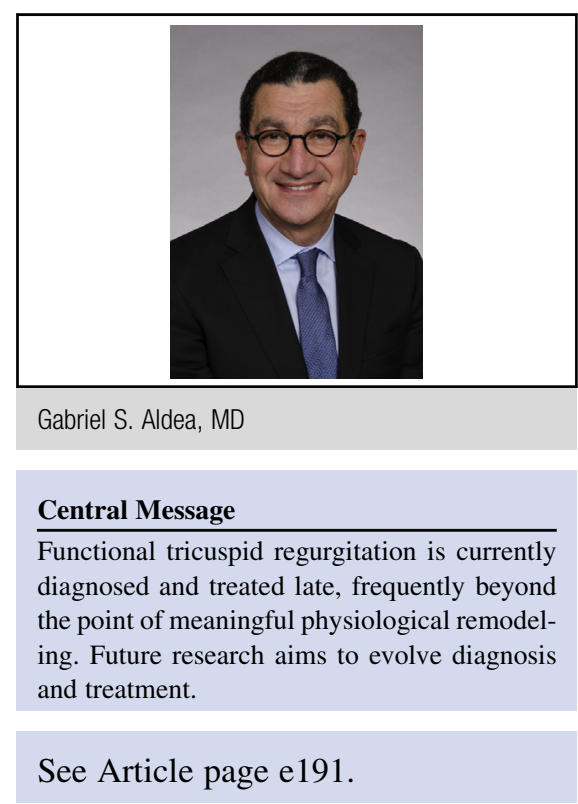

This recent publication by Malinowski and colleagues ${ }^{10}$ represents another facet of their ongoing efforts to better assess and define TV anatomy, function, and acute pathology. ${ }^{11}$ In this specific publication, the impact of different annuloplasty ring characteristics on TV function was assessed with a stated goal of better device design in the hope of promoting TV repair durability. Using a known model of ovine, acute, normal (non-dilated) hearts and sonomicrometry and pressure transducers, the authors were able to demonstrate that all 3 annuloplasty rings reduce the diastolic TV annular area (by nearly one-half). In comparison with flexible Duran and Tri-Ad rings, the Contour rigid non-planar ring was better at maintaining the 3-dimensional annular (ellipsoid) saddle-shape, reducing the magnitude of cardiac strain RV wall stress and remodeling, and increasing leaflet coaptation but doing so at the expense of reducing annular "physiological" contraction. Maintaining annular shape and height may be associated with decreased leaflet strain and stress and enhanced repair durability but at the potential risk of a greater incidence of ring dehiscence. The authors fully acknowledge that clinical (chronic) TR significantly impacts TV annular contraction, dilation, flattening, and strain. Chronic pathologic changes limit extrapolation of these findings to common and varied clinical scenarios. It is difficult to draw conclusions of 
whether these varied ring characteristics will differentially impact RV stress or longer-term outcomes in different temporal phases, severity, and anatomic presentations of clinically relevant $\mathrm{TR}$.

It is evident that our understandings of TV physiology and pathology are currently still limited. Most significantly, FTR is currently diagnosed late, frequently beyond the point of meaningful physiological remodeling of associated profound accompanying RV or right atrial pathology. It is unclear whether current therapies (both open surgical and emerging catheter-based) are sufficiently tailored and complete, or are simply too little too late, to effectively impact long-term survival. We hope that future research will lead to earlier, more nuanced pathophysiologic staging and diagnoses and also lead to more timely, targeted, and effective therapies.

\section{References}

1. Dietz MF, Prihadi EA, van der Bijl P, Goedemans L, Mertens BJA, Gursoy E, et al. Prognostic implications of right ventricular remodeling and function in patients with significant secondary tricuspid regurgitation. Circulation. 2019;140: 836-45.

2. Chang CC, Veen KM, Hahn RT, Bogers AJJC, Latib A, Oei FBS, et al. Uncertainties and challenges in surgical and transcatheter tricuspid valve therapy: a state-of-the-art expert review. Eur Heart J. September 11, 2019 [Epub ahead of print].

3. Nath J, Foster E, Heidenreich PA. Impact of tricuspid regurgitation on long-term survival. J Am Coll Cardiol. 2004;43:405-9.

4. Wang N, Fulcher J, Abeysuryia N, McGrady M, Wilcox I, Celermajer D, et al. Tricuspid regurgitation is associated with increased mortality independently of pulmonary pressures and right heart failure: a systematic review and meta-analysis. Eur Heart J. 2019;40:476-84.

5. Topilsky Y, Inohosa JM, Benfari G, Vaturi O, Maltais S, Michelena H, et al. Clinical presentations and outcomes of tricuspid regurgitation in patients with systolic dysfunction. Eur Heart J. 2018;39:3584-92.

6. Brescia AA, Ward ST, Watt TMF, Rosenbloom LM, Baker M, Khan S, et al; Michigan Mitral Research Group (MMRG). Outcomes of guideline-directed concomitant annuloplasty for functional tricuspid regurgitation. Ann Thorac Surg. August 31, 2019 [Epub ahead of print].

7. Badhwar V, Rankin JS, He M, Jacobs JP, Furnary AP, Fazzalari FL, et al. Performing concomitant tricuspid valve repair at the time of mitral valve operations is not associated with increased operative mortality. Ann Thorac Surg. 2017;103: 587-93.

8. Lee JW, Song JM, Park JP, Lee JW, Kang DH, Song JK. Long-term prognosis of isolated significant tricuspid regurgitation. Circ J. 2010;74:375-80.

9. Zack CJ, Fender EA, Chandrashekar P, Reddy YNV, Bennett CE, Stulak JM, et al. National trends and outcomes in isolated tricuspid valve surgery. J Am Coll Cardiol. 2017;70:2953-60.

10. Malinowski M, Jazwiec T, Quay N, Goehler M, Rausch MK, Timet TA. The influence of tricuspid annuloplasty prostheses on ovine annular geometry and kinematics. J Thorac Cardiovasc Surg. 2021;161:e191-207.

11. Jazwiec T, Malinowski M, Bush J, Goehler M, Quay N, Parker J, et al. Right ventricular free wall stress after tricuspid valve annuloplasty in acute ovine right heart failure. J Thorac Cardiovasc Surg. 2019;158:759-68. 